\title{
Analytic Evaluation of Shared-Memory Architectures
}

\author{
Daniel J. Sorin, Member, IEEE, Jonathan L. Lemon, Derek L. Eager, and \\ Mary K. Vernon, Member, IEEE Computer Society
}

\begin{abstract}
This paper develops and validates an efficient analytical model for evaluating the performance of shared memory architectures with ILP processors. First, we instrument the SimOS simulator to measure the parameters for such a model and we find a surprisingly high degree of processor memory request heterogeneity in the workloads. Examining the model parameters provides insight into application behaviors and how they interact with the system. Second, we create a model that captures such heterogeneous processor behavior, which is important for analyzing memory system design tradeoffs. Highly bursty memory request traffic and lock contention are also modeled in a significantly more robust way than in previous work. With these features, the model is applicable to a wide range of architectures and applications. Although the features increase the model complexity, it is a useful design tool because the size of the model input parameter set remains manageable, and the model is still several orders of magnitude quicker to solve than detailed simulation. Validation results show that the model is highly accurate, producing heterogeneous per processor throughputs that are generally within 5 percent and, for the workloads validated, always within 13 percent of the values measured by detailed simulation with SimOS. Several examples illustrate applications of the model to studying architectural design issues and the interactions between the architecture and the application workloads.
\end{abstract}

Index Terms-Analytical model, shared memory multiprocessor, heterogeneity, performance evaluation, mean value analysis.

\section{INTRODUCTION}

OMPUTER architects traditionally use detailed simulation to evaluate architecture performance tradeoffs. Detailed simulation of parallel architectures with complex modern processors usually entails a cycle-by-cycle simulation of each processor that precisely captures significant behavior, such as out-of-order instruction issue and speculative instruction execution, that can greatly affect system performance [13]. Detailed simulation, however, is time-consuming. For example, detailed simulation of an 8-processor shared memory architecture, running a single parallel FFT code with a small input data set, can take hours on a Sun UltraSPARC system, even though only seconds of the system execution time are actually simulated.

To design a given memory system architecture, one would like to evaluate alternative memory system architectures for dozens, if not hundreds, of commercial applications and workloads that might be expected to run on the system. Thus, more efficient evaluation methods that can aid in culling the system design space are highly desirable. Analytical models offer the possibility of

- D.J. Sorin is with the Department of Electrical and Computer Engineering, Duke University, Box 90291, Durham, NC 27708.

E-mail: sorin@ee.duke.edu.

- J.L. Lemon is with Cisco Systems, 117 W. Chicago St., Stoughton, WI, 53589. E-mail: jlemon@flugsvamp.com.

- D.L. Eager is with the University of Saskatchewan, Room 1c112, Engineering Building, 57 Campus Drive, Saskatoon, SK S7N 5A9. E-mail: eager@cs.usask.ca.

- M.K. Vernon, 1210 W. Dayton Street, Madison, WI 53706. E-mail:vernon@cs.wisc.edu.

Manuscript received 22 Feb. 2000; revised 13 July 2001; accepted 9 Aug. 2002.

For information on obtaining reprints of this article, please send e-mail to: tpds@computer.org, and reference IEEECS Log Number 111556. efficiently computing performance estimates that can be useful in identifying the most promising regions of the architectural design space, which can then be explored more fully using the detailed simulation approach. The key issue is devising an analytical model that is sufficiently accurate for this purpose, over the range of workloads of interest. If such a model can be constructed, it also offers the opportunity to explore how the memory system architecture performs for hypothetical changes in the memory request behavior of the executing workload. Experienced system architects may be interested in exploring such issues, which is difficult to do with simulation of specific benchmarks.

Three recent papers have developed analytical models that contain some of the significant features of complex, modern, and shared memory multiprocessor architectures [3], [21], [17]. Of these models, the previous "SM-ILP" model [17] is the only model that 1) includes the impact of instruction window size and dependences between memory accesses, which cause a processor to block after a dynamically changing number of memory requests, and 2) has been validated against simulations of applications running on a parallel shared memory architecture. The model has a number of significant features. First, it is based on a relatively small set of input parameters that are sensitive to changes in the processor and associated cache architecture, but are insensitive to changes in the rest of the memory system architecture. Second, the model captures the key characteristics of a complex modern processor architecture which are important for memory system design, such as speculative memory requests and complex processor blocking behavior. Third, the SM-ILP model produces results for each alternative memory system architecture in a few seconds and these results were shown to predict processor 
throughput, measured in instructions per cycle (IPC), within 1-12 percent of the detailed simulation estimates for several Splash-2 applications [23] running on the RSIM architecture [12].

This paper extends the SM-ILP model in the following ways in order to create a complete model of system behavior:

- Measurements of several SPMD applications running on RSIM showed that each processor is statistically identical with respect to memory request behavior and that, for each processor, its remote memory requests are approximately equally likely to visit each of the remote memory modules [17]. This paper measures the parameters for some of the same SPMD applications as well as other Splash-2 applications using SimOS, which has been used in several previous architecture, OS, and workload studies [19], [20], [4] and includes the operating system workload as well as the application workload. The SimOS measurements show that, although the SGI IRIX operating system executes uniformly across all of the processors, both the SPMD and the other Splash-2 applications have highly heterogeneous memory request behavior. Measured parameters provided in this paper illustrate the types of heterogeneity that occur in the workloads that are simulated using SimOS. More detailed measures are provided to understand the several causes of the observed heterogeneity.

- $\quad$ The new model provides parameters for specifying heterogeneous, as well as homogeneous, memory request behavior. That is, each processor can have a different mean time between memory requests, distribution of the number of outstanding requests when the processor blocks, and so forth. Moreover, the memory requests from each processor can have a different distribution of destinations for requests to remote nodes. Since heterogeneous memory request behavior can have a disproportionate impact on system throughput due to nonlinear queuing effects in the memory system, it is important for a model that supports memory system design to capture such behavior. The extensions to the SM-ILP model that are needed to solve the heterogeneous system models are relatively straightforward. One key open question addressed in this work is whether the model remains tractable, both from a programming effort standpoint and a solution-time/convergence standpoint, when the memory request heterogeneity is represented. Another key open question is how well the heterogeneous model validates with respect to individual processor throughput estimates and with respect to estimated mean queuing delays in the memory system.

- $\quad$ The SM-ILP parameter measurements revealed that memory requests from modern processors with nonblocking caches are highly bursty and that this burstiness can have a significant impact on the queuing times in the memory systems [17]. The SMILP model used a simple analytic approach to represent the performance impact of the bursty requests which was reasonably accurate for estimating overall system throughput, but it overestimated processor utilization and underestimated bus waiting times in the RSIM architecture. Recent work [5] develops new analytic methods that more accurately estimate server utilizations and mean waiting times in simple two-queue networks with bursty departures from one of the queues. The key issues addressed in this paper are how to parameterize the new analytic methods in [5] for the context of bursty memory requests in shared memory system architectures and whether the new methods are accurate in this more complex context.

- The SM-ILP model computes total application running time from measured average lock waiting times as well as analytic estimates of the processing rate when the processors are not waiting for locks. Since lock contention delays are affected by delays in the memory system, the average lock waiting time input parameter is, in general, dependent on the output values of the model. This paper develops a submodel that accurately estimates mean lock access delays from fundamental input parameters that are independent of changes in the memory system architecture (below the processor cache hierarchy).

With the above extensions, the model presented and validated in this paper is significantly more complete than the SM-ILP model. Moreover, the input parameters themselves provide new understanding of application behavior. While modeling the additional behaviors increases the model's size and complexity over that of the SM-ILP model, the model solution time is still on the order of a couple of seconds. The number of input parameters is increased, but is still manageable.

The model extensions developed in this paper could easily be applied to the RSIM architecture. The new application to the system architecture simulated by SimOS has two significant benefits. First, the new application tests the robustness of the basic analytic approach for a significant change in the memory system architecture, including a different memory consistency model. Second, the measured input parameters for the model show that the SimOS workloads, which include operating system processing, have quite different behavior than RSIM workloads with respect to the memory system.

Validations in this paper show that the new model predicts heterogeneous processor performance that agrees with detailed SimOS estimates for a set of benchmark applications running on the SimOS architecture. The percentage difference between the throughput estimates computed by the model and the throughput reported by SimOS for each processor is typically within 5 percent and always less than 13 percent over the workloads studied in this work. The validation results and example model applications also show that modeling heterogeneity is important for achieving high model accuracy. Thus, this capability is essential both to achieving wider applicability of the model and for increasing confidence in using the model to find the promising regions of the memory system architecture design space that should be investigated using detailed simulation.

Three examples are provided to illustrate the use of the new model. One example illustrates the use of the model to evaluate alternative memory system designs under a heterogeneous workload. The two other examples provide estimates of the (maximum) performance gains that can be achieved if applications are "tuned" to remove the heterogeneity that is observed in the measured parameters for the applications.

Like the previous analytic model, the new model input parameters are derived from a detailed simulation of an 


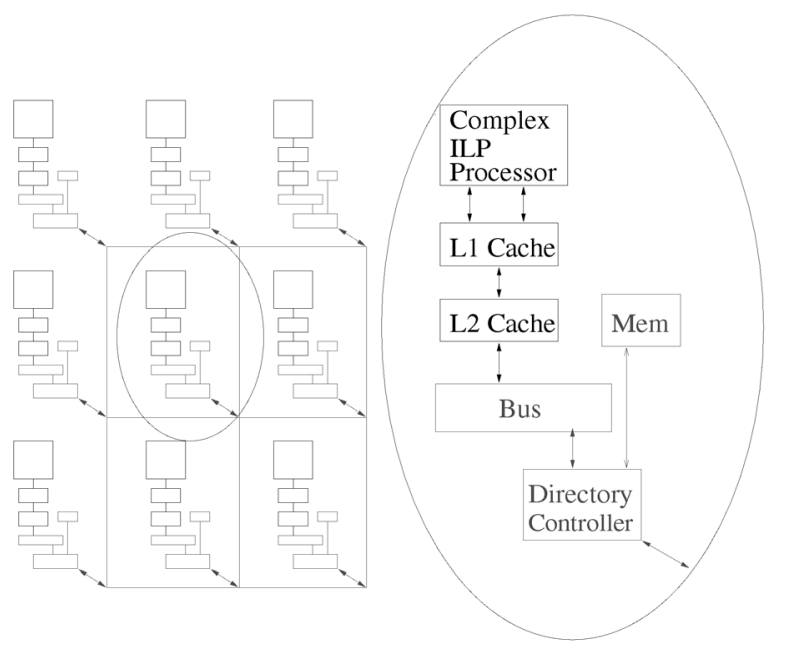

Fig. 1. System architecture.

application or workload running on a given parallel processor architecture. However, the model input parameters have been carefully chosen so as to be insensitive to large changes in the memory system latency below the processor cache hierarchy. Thus, as shown in [17], the analytic model can accurately predict system performance when various memory system components below the processor cache hierarchy are modified. Consequently, the analytic model can be used to quickly cull the design space for this part of the memory system for both measured workload parameters and hypothetical variations in the measured workload parameters, thereby greatly extending the region of the design space that can be evaluated as well as reducing the size of the design space that needs to be explored using simulation. More detailed exploration of the promising regions of the design space can be performed using full system simulators, detailed models of caches (e.g., [2], [15], [22]), and/or statistical simulation approaches [11] to obtain additional insights.

The rest of this paper is organized as follows: Section 2 describes the system that will be modeled. Section 3 explains the model parameters and provides measured application parameters that illustrate the types of heterogeneity that occur in the SimOS benchmarks. Section 4 discusses the model and develops new modeling approaches for bursty traffic and synchronization in shared memory multiprocessors. Section 5 presents the model validations and Section 6 discusses applications of the model. Finally, Section 7 summarizes the paper and discusses future research.

\section{System Architecture}

The system architecture of interest in this paper is the architecture modeled by SimOS [14]. This architecture, which is similar to that of the Stanford FLASH [7] and the SGI Origin [8], is a cache-coherent, sequentially consistent shared-memory multiprocessor system, as shown in Fig. 1. Differences between this architecture and the RSIM architecture [12] previously modeled in the SM-ILP model are pointed out in the description of the SimOS architecture below.

The MIPS R10000 processor, modeled by SimOS's MXS simulator, is an aggressive implementation of sequential consistency (SC) that exploits instruction level parallelism using multiple functional units, out-of-order execution, nonblocking loads, and speculative execution. Instructions
TABLE 1

System Architecture Parameters

\begin{tabular}{|l|l|r|}
\hline parameter & description & value \\
\hline$N$ & number of nodes & \\
$m$ & memory modules per node & 1 \\
$M_{h w}$ & number of MSHRs & 8 \\
$S_{b u s}$ & bus latency & 15 \\
$S_{D C}$ & short directory controller (DC) latency & 5 \\
$S_{D C_{l o n g}}$ & long directory controller latency & 20 \\
$S_{n e t}$ & average network traversal latency & 30 \\
\hline
\end{tabular}

are fetched into the instruction window and they are issued to the functional units after all of their input data dependences are satisfied. Speculative execution is used for (temporarily) unresolved control dependences for up to four branch instructions. The instructions are fetched into, and retired from, the window in program order, but they may be issued to the functional units out of program order.

An instruction can retire from the instruction window only after it completes execution. A key implication of this requirement is that, when a load reaches the top of the instruction window, retirement must stall if the data has not yet returned. SimOS differs from RSIM in that, since SimOS models a sequentially consistent architecture, stores in SimOS only issue to the memory system when they reach the top of the instruction window.

The caches use miss status holding registers (MSHRs) to track the status of all outstanding misses [6]. Misses to the same cache line are coalesced in the MSHRs; only one memory request is generated for such coalesced misses.

As shown in Fig. 1, all traffic to or from a remote node goes through the directory controller (DC). SimOS models the memory bus and the interconnection network using fixed latencies that account for service time as well as estimated contention delay. In contrast to the RSIM architecture, traffic into the node that only requires accessing the directory and memory does not require use of the bus. SimOS also differs from RSIM in that it does not model a separate network interface since the DC serves that purpose.

Cache coherence is maintained by a fairly standard three-state (MSI) directory-based invalidation protocol. Unlike the RSIM architecture, cache-to-cache transfers require four hops instead of three; the home node is responsible for collecting invalidations before acknowledging a request for exclusive permission.

\section{Parameters}

In this section, we describe the input parameters for the model. These parameters include the system architecture parameters and the application parameters. Then, we show that the application parameters exhibit heterogeneity across the processors and we explain several sources of this heterogeneity.

\subsection{System Architecture Parameters}

Table 1 defines the system architecture parameters, including the values that are used in the validation experiments in Section 5. Latencies are in units of CPU cycles for the $200 \mathrm{MHz}$ R10000 processor. Note that memory access is overlapped with directory access; thus, there is just one parameter for that access latency, $S_{D C_{l o n g}}$. 
TABLE 2

Application Parameters

\begin{tabular}{|c|c|}
\hline Parameter & Description \\
\hline$\tau$ & $\begin{array}{l}\text { Average time between read, write, or upgrade requests to memory, not count- } \\
\text { ing the time when the processor is completely stalled or is spin-waiting on a } \\
\text { synchronization event }\end{array}$ \\
\hline$C V_{\tau}$ & Coefficient of Variation of $\tau$ \\
\hline$f_{M}$ & $\begin{array}{l}\text { Fraction of processor stalls that occur with } M=1,2, \ldots \text { outstanding requests } \\
\text { in the MSHRs }\end{array}$ \\
\hline$P_{\text {read }}, P_{\text {write }}, P_{\text {upgrade }}$ & Probability that a memory request is a read, write, or upgrade \\
\hline & Probability that a read or write request causes a writeback of a cache block \\
\hline$P_{L \mid x}$ & $\begin{array}{l}\text { Probability directory is local for a type } x \text { transaction; } \\
x=\text { read, write, upgrade, writeback }\end{array}$ \\
\hline$P_{M \mid x, y}$ & $\begin{array}{l}\text { Probability home memory can supply the data for a type } x, y \text { request; } \\
x=\text { read, write; } y=\text { local home, remote home }\end{array}$ \\
\hline$P_{4 h o p \mid x \& \text { not-memory }}$ & $\begin{array}{l}\text { Probability that a request of type } x \text { to a remote home is forwarded to a cache } \\
\text { at a third node; } \\
x=\text { read,write }\end{array}$ \\
\hline$X$ & Average number of invalidates caused by a write or upgrade to a clean line \\
\hline
\end{tabular}

\subsection{Application Parameters}

The application parameters for a given processor are summarized in Table 2. These parameters characterize the memory request behavior (between any two barriers) of an application running on the architecture. For a homogeneous model, the parameter values are the same at all processors, but this will not be true of the heterogeneous model. The first three parameters characterize the rate of requests to the memory system, the burstiness in the memory interrequest times, and the number of outstanding requests when the processor blocks due to a memory request that cannot be retired. Note that the distribution $f_{M}$ accounts for the impact of speculative (as well as nonspeculative) memory requests on processor blocking behavior. The rest of the parameters characterize the types of requests that are being issued to the memory system. Sorin et al. observe that these input parameters are sensitive to instruction window size, processor architecture, organization and size of the processor cache hierarchy, and various aspects of the application code and compiler, but are relatively insensitive to memory system latencies below the processor cache hierarchy [17].

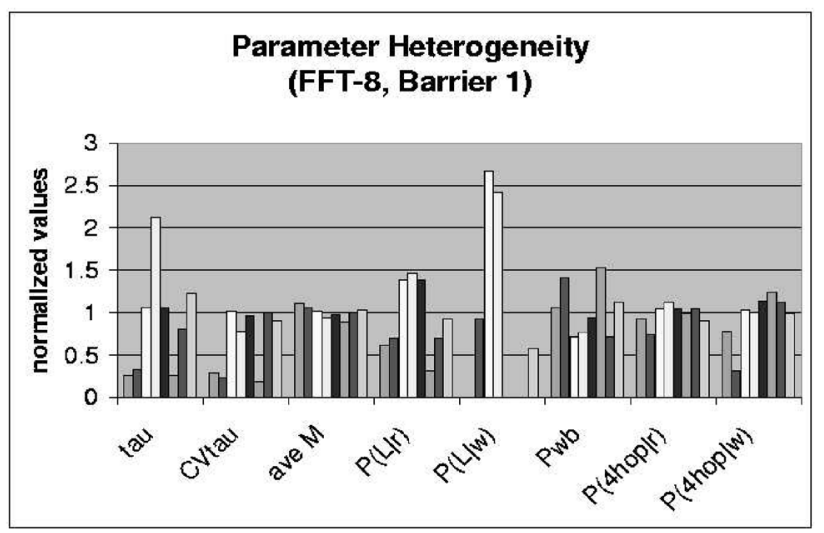

Fig. 2. Parameter heterogeneity of FFT-8, barrier 1.

\subsection{Heterogeneity in Application Parameters}

The homogeneous SM-ILP model assumes that all processors have statistically similar application behavior with respect to the memory system and that each processor's local/remote memory accesses are uniformly distributed across the local/remote memory modules. In the homogeneous model, each processor has the same input parameters, as shown in Table 2, and no input parameters are needed for frequencies of access to each memory module.

Fig. 2, Fig. 3, and Fig. 4 illustrate the processor heterogeneity in several key parameters for particular barriers (i.e., interbarrier phases) of a few SPLASH-2 benchmarks [23], as measured by SimOS. The benchmark name is followed by the number of processors, e.g., FFT-8 is an eight processor run of the FFT benchmark. For each input parameter shown, the eight bars represent the values of that parameter for each of the eight processors, divided by the value of that parameter when measured over all eight processors. The heterogeneity in various measures for particular barriers is also summarized in Table 3.

The degree of parameter heterogeneity, such as in the measures of $\tau$ and $P(L \mid w)$ in the figures and Table 3, is

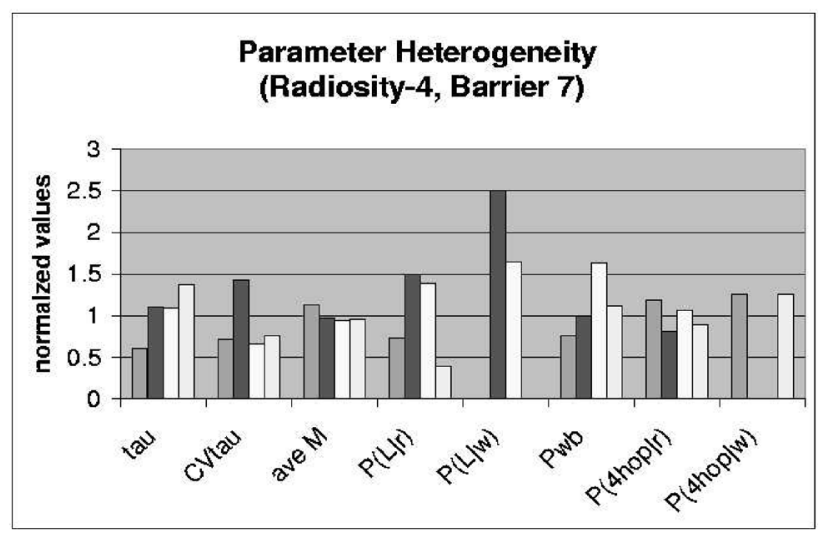

Fig. 3. Parameter heterogeneity of Radiosity-4, barrier 7 . 


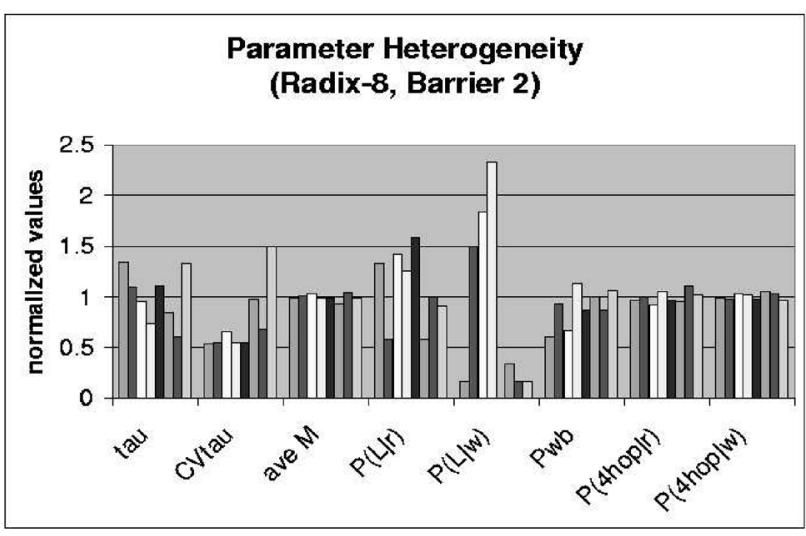

Fig. 4. Parameter heterogeneity of Radix-8, barrier 2.

perhaps higher than might be expected. Some (irregular) applications, such as Radiosity, are inherently heterogeneous and, thus, the per processor memory request measures simply quantify the degree of memory request heterogeneity that occurs in those applications. However, as can be seen from the figures and the table, significant memory request heterogeneity is also present for SPMD applications, such as FFT and Radix, that are generally thought to be homogeneous and were observed to have homogeneous memory request behavior in RSIM [12]. It is particularly surprising to observe the heterogeneity in the statistics for systems with small numbers of processors, and it is relevant since SMPs with four or eight processors are prevalent in industry. We are not aware of any prior work that shows that SPMD applications have a high degree of statistical heterogeneity.

In an attempt to determine the source of the processor memory request heterogeneity for these SPMD applications on SimOS, we separated the statistics into application and kernel statistics for each processor. For example, the number of memory requests per processor for the transpose phase (barrier 2) of FFT-4 is shown in Table 4 . As expected, the number of memory requests that are issued when a processor is executing the application are quite homogeneous across the processors, with a coefficient of variation of 0.02 . What is perhaps surprising is both the high numbers of memory requests that are issued in kernel mode (larger, in fact, than the numbers of application requests) as well as the heterogeneity in this number across processors, as shown by a coefficient of variation of 0.27 . For the SPMD applications, other parameters like $C V_{\tau}$ exhibit similar heterogeneity in the kernel while remaining homogeneous in the application. ${ }^{1}$

A key point is that the kernel executes uniformly across the processors in the SimOS architecture and, thus, the kernel memory request statistics are fairly homogeneous across the processors if measured over a long time interval, such as the execution time for the entire application. However, the memory request heterogeneity observed in the measured intervals between each barrier will impact memory system performance and, therefore,

1. Note that, in Table 4, the number of memory requests appears to be correlated with the processor number, but this correlation is just coincidental and did not occur with any higher than random frequency in the results that we obtained for different barriers in the FFT application and for different applications.
TABLE 3

Examples of Parameter Heterogeneity

\begin{tabular}{|l|l|l|r|r|r|r|}
\hline benchmark & barrier & parameter & mean & $\min$ & $\max$ & CV \\
\hline FFT-8 & 2 & $\tau$ & 60 & 42 & 78 & .15 \\
& 3 & $P(L \mid u)$ & .13 & .01 & .30 & .73 \\
\hline Radiosity-4 & 3 & $\tau$ & 78 & 48 & 122 & .35 \\
& 7 & $P(4 h o p \mid r)$ & .66 & .53 & .78 & .14 \\
\hline Radix-8 & 1 & $P(L \mid w)$ & .12 & .01 & .33 & 1.17 \\
& 3 & $\tau$ & 103 & 31 & 210 & .45 \\
& 4 & $P(L \mid r)$ & .13 & .04 & .25 & .47 \\
\hline
\end{tabular}

the heterogeneity in this timeframe must be measured and represented in the inputs to the model.

Beyond the heterogeneity inherent in some applications or caused by kernel behavior, some applications can exhibit heterogeneous behavior if they have not been "tuned" to run on a particular architecture and runtime system, which occurs frequently in practice. Heterogeneity in the measured model input parameters can point to the need for such tuning and even indicate what types of tuning are needed. For example, in barrier 1 of FFT-8, three processors have small relative values of $\tau$ when the application is executing, indicating that they have especially high level 2 cache miss rates. Those same processors (and one additional processor) have a relatively low probability of local memory access for read and/or write requests. Thus, examining the data layout or comparing the code that runs on those three processors against the code that runs on the other five processors, looking for differences that might cause these effects, may lead to some insight about how to improve performance. Similarly, the heterogeneity in the probability that a write request is local for Radix suggests that data layout should be examined for that application as well.

In general, although there may be intuition that particular applications will exhibit heterogeneous behavior of some form, intuition alone is generally insufficient to estimate the magnitude of the heterogeneity in particular statistics of interest, its magnitude relative to the heterogeneity induced by kernel activity, or the extent to which performance might be improved by particular types of application tuning. Measurements of heterogeneity and the use of models that capture its performance impacts can provide answers to such questions.

The figures and the table indicate that heterogeneity occurs in practice for every model input parameter. Two parameters, though, have notably less extreme heterogeneity: 1) the average of the $f_{M}$ distribution (i.e., the average number of memory requests that are outstanding when the processor blocks because a load or store cannot be retired) and 2) the probability that a remote read request for a dirty

TABLE 4

Memory Requests for FFT-4 Barrier 2

\begin{tabular}{|l|l|l|l|l|}
\hline & cpu 0 & cpu 1 & cpu 2 & cpu 3 \\
\hline overall & 461 & 613 & 781 & 787 \\
\hline application & 216 & 219 & 231 & 219 \\
kernel & 245 & 394 & 550 & 568 \\
\hline
\end{tabular}


block requires invalidating or downgrading the line in a cache at another remote node $\left(P_{4 h o p \mid r}\right)$. However, system performance can be sensitive to the values of these parameters. Thus, the model extensions for processor heterogeneity will allow each processor to have its own value for each of the input parameters.

\subsection{Methodology for Obtaining the Application Parameters}

As mentioned in Section 3.2, previous work has shown that the homogeneous model input parameters are, to first order, insensitive to changes in memory system latency below the processor cache hierarchy. For the new model developed in this paper, we use the same input parameters for each processor, but allow each processor to have a different value for each parameter. Thus, these input parameters will also be insensitive (to first order) to changes in the memory system latencies below the processor-cache hierarchy. One question is whether these parameters are sufficient for accurately computing processor throughputs and mean delays in the memory system for heterogeneous workloads. This question will be investigated by comparing the estimates against the performance measures that are given by SimOS.

The set of parameters for a given application/workload of interest executing on a given processor and cache architecture of interest are obtained through simulation of the application on a single memory and interconnection network architecture (e.g., an idealized constant latency interconnect) with memory access latencies that are within a small constant factor of the latencies in the memory architectures to be evaluated with the model. Currently, as shown in [17], the most accurate way to estimate these parameters is to use the detailed simulator (e.g., SimOS) that will be used to further evaluate the promising memory system architectures identified by the analytic model. ${ }^{2}$

A key point in this methodology is that the detailed simulator is run once for each workload and a given processor/cache architecture to obtain the analytic model parameters. The analytic model is then used to evaluate many candidate memory and interconnection network architectures, as illustrated in Section 6 of the paper. The detailed simulator is then used to evaluate further details of the most promising memory/interconnect architectures. Because the detailed simulator is needed for detailed analysis of the more promising architectures, the one run needed per application to obtain the parameters for the analytic model does not add significantly to the total time needed to evaluate the architectures. Conversely, the analytic model is more easily modified for alternative memory/interconnect architectures than the simulator (because the analytic model is more abstract and the equations each have one of several possible forms) and it can significantly speed up evaluation of the alternative architectures.

2. Faster methods might be developed to obtain some of the parameters, as was investigated for the SM-ILP model, but those parameters have, so far, been less accurate than the parameters from the detailed simulator and improvements in the faster simulation methods are beyond the scope of this paper.

\section{Analytical Model}

In this section, we develop the extended analytical model for the SimOS architecture. The principal output measure computed by the model is the system throughput, measured in instructions retired per cycle (IPC). This throughput, as well as mean waiting time and utilization of each memory system resource, is computed as a function of the input parameters that characterize the workload and the memory architecture.

For simplicity, in the exposition of the model equations, we first present the homogeneous model in Section 4.1 and then present the extensions for the heterogeneous model in Section 4.2. In Section 4.3, we develop the techniques for modeling bursty memory traffic and lock contention.

\subsection{Homogeneous Model}

As in the previous SM-ILP model [17], we develop a customized Approximate Mean Value Analysis (AMVA) model of homogeneous workloads running on the SimOS architecture. Our experience is that, as claimed in that paper, it is not difficult to modify the basic AMVA equations in the previous model [18] for other shared memory multiprocessor architectures. The most significant issue in developing the new model was how to model the different memory consistency model in the SimOS architecture. Unlike the SM-ILP model which iterated between two submodels to account for two types of processor blocking behavior in the release consistent (RC) RSIM architecture, the model of the sequentially consistent (SC) SimOS architecture developed below is a single model that accounts for all types of blocking behavior.

The processor and cache subsystem are modeled as a black box that-when not completely stalled-issues memory requests at a given rate $(1 / \tau)$ and with a given coefficient of variation in interrequest times $\left(C V_{\tau}\right)$. The model computes the overall mean system residence time for a memory request, including mean delays and service times at the directory controllers (DCs), split-transaction memory buses, and in the interconnection network.

For readability, we have adopted the following notation of subscripts and superscripts for the variables in the model. The resource is always the first subscript on a variable, whether it is mean residence time $(R)$, mean waiting time $(W)$, mean utilization $(U)$, or mean service time $(S)$. For example, $R_{d c}$ is the mean residence time at the directory controller. For many terms, there is a subscript of loc or rem to indicate whether the resource is at the local node for a given processor or a remote node. The subscript variable $y$ denotes the transaction type (such as read or write). We first present the equations for the case that $f_{M}=1$ for a particular but arbitrary value of $M$ less than or equal to the number of MSHRs. In this case, each processor has $M$ customers that each alternately visit the processor for average time $\tau$ and then visit various resources in the memory system, reflecting (statistically) the memory request behavior from the processor. Later, we discuss how to model the more general distribution for $f_{M}$.

The following equation is for the total mean residence time of a customer for one cycle from the processor, through the memory system, and back to the processor. This includes the mean residence times at the processor, buses (both local and remote), network, and directory controllers, 


$$
R=R_{p e}+R_{b u s}+R_{n e t}+R_{d c} \text {. }
$$

Each of these terms is derived from lower level equations. For example, the mean residence time at the directory controllers is equal to the sum of the mean residence time at the local DC and at the remote DCs,

$$
R_{d c}=R_{d c_{\text {loc }}}+R_{d c_{\text {rem }}} \text {. }
$$

The mean residence time at the local (remote) DC is equal to the sum of the weighted mean residence time for each transaction type $y$ at the local (remote) DC, weighted by the probability that the transaction is of type $y$,

$$
\begin{aligned}
R_{d c_{\text {loc }}} & =\sum_{y} R_{d c_{l o c, y},} \\
R_{d c_{\text {rem }}} & =\sum_{y} R_{d c_{\text {rem }, y} .} .
\end{aligned}
$$

The weighted mean residence time of a transaction of type $y$ at the local (remote) DC is equal to the probability of transaction $y$ times the average number of visits the type $y$ transaction makes to the local (remote) DC $\left(V_{\text {dir } r_{\text {locy }}}\right)$ times the sum of the waiting time at the local (remote) $\mathrm{DC}\left(W_{d c_{\text {loc }}}\right)$ and the service time at a DC,

$$
\begin{gathered}
R_{d c_{l o c, y}}=P_{y} V_{d c_{l o c y}}\left(W_{d c_{l o c}}+S_{d c}\right), \\
R_{d c_{\text {rem }, y}}=P_{y} V_{d c_{\text {rem }}}\left(W_{d c_{\text {rem }}}+S_{d c}\right) .
\end{gathered}
$$

Note that the average number of visits to the local (remote) DC is computed for the transaction type using the other probabilities given in Table 2.

All of the terms in the above equations are inputs, except the waiting times. $W_{d c_{l o c}}$ consists of the waiting time at the local DC due to requests from the local node $\left(W_{d c_{l o c}}^{l o c}\right)$ and due to requests from remote nodes $\left(W_{d c_{l o c}}^{r e m}\right)$,

$$
W_{d c_{l o c}}=W_{d c_{l o c}}^{l o c}+W_{d c_{l o c}}^{r e m} .
$$

The waiting time at the local DC due to requests from the local node equals the sum of the waiting times over all transaction types $y$ that cause waiting,

$$
\begin{gathered}
W_{d c_{l o c}}^{l o c}=\sum_{y} W_{d c_{l o c}}^{l o c, y}, \\
W_{d c_{l o c}}^{r e m}=\sum_{y} W_{d c_{l o c}^{r e m, y}}^{r e} .
\end{gathered}
$$

$W_{d c_{r e m}}$ consists of the waiting time due to remote customers that are not from that remote node $\left(W_{d c_{\text {rem }}}^{\text {others }}\right)$ and those that are from that remote node $\left(W_{d i i_{r e m}}^{r e m}\right)$,

$$
\begin{gathered}
W_{d c_{r e m}}=W_{d c_{r e m}}^{o t h e r s}+W_{d c_{r e m}}^{\text {rem }}, \\
W_{d c_{r e m}}^{\text {others }}=\sum_{y} W_{d c_{r e m}, \text { others }, y} \\
W_{d c_{r e m}}^{\text {rem }}=\sum_{y} W_{d c_{r e m}}^{\text {rem, } y}
\end{gathered}
$$

The following equations are for the mean waiting times due to waiting for specific transaction types. For example,
$W_{d c_{l o c}}^{l o c, y}$ is the mean waiting time at the local DC due to local requests of transaction type $y$. Mean waiting time for a single other customer equals $\frac{R_{d c_{l o c, y}}}{R}-U_{d c_{l o c, y}}$ (the probability that a customer is in the queue but not in service) times the service time plus $U_{d c_{l o c, y}}$ (the probability that a customer is in service) times the mean residual life of a customer in service. Therefore, to get the total mean waiting time, we multiply by the number of local customers who could cause an arriving local customer to wait, $M-1$.

$$
\begin{aligned}
W_{d c_{l o c}}^{l o c, y}= & (M-1)\left[\left(\frac{R_{d c_{l o c, y}}}{R}-U_{d c_{l o c, y}}\right) S_{d c}+U_{d c_{l o c, y}}\left(\frac{S_{d c}}{2}\right)\right] \\
W_{d c_{l o c}}^{r e m, y}= & M(N-1)\left[\left(\frac{R_{d c_{r e m, y}, y}}{R}-U_{d c_{r e m, y}, y}\right) S_{d c}+U_{d c_{r e m, y},}\left(\frac{S_{d c}}{2}\right)\right] \\
W_{d c_{r e m}}^{o t h e r s, y}= & {[(M-1)+M(N-2)]\left[\left(\frac{R_{d c_{r e m, y}}}{R}-U_{d c_{r e m, y}}\right) S_{d c}\right.} \\
& \left.+\left(U_{d c_{r e m, y}}\right)\left(\frac{S_{d c}}{2}\right)\right] \\
W_{d c_{r e m}}^{r e m, y}= & M\left[\left(\frac{R_{d c_{l o c, y}}}{R}-U_{d c_{l o c, y}}\right) S_{d c}+\left(U_{d c_{l o c, y}}\right)\left(\frac{S_{d c}}{2}\right)\right] .
\end{aligned}
$$

Last, we have the utilization equations. The first equation is the mean utilization of a DC by a local customer, and the second equation is the mean utilization of a DC by a remote customer,

$$
\begin{gathered}
U_{d c_{l o c, y}}=\frac{P_{y}}{R}\left(V_{d c_{l o c, y}} S_{d c}\right), \\
U_{d c_{\text {rem }, y}}=\frac{P_{y}}{R}\left(V_{d c_{r e m, y}} S_{d c}\right) .
\end{gathered}
$$

A key question in developing the analytic model is how to compute throughput as a function of the dynamically changing number of outstanding memory requests that can be issued before the processor must stall waiting for data to return from memory. The SM-ILP model resolved this issue by solving the model for each value of $M$ and then taking a weighted average of the results. As will be explained in the next section, a different solution will be necessary for a heterogeneous model.

\subsection{Modeling Heterogeneity}

Given that 1 ) processor heterogeneity (and memory access nonuniformity) occur quite frequently in practice and 2) the heterogeneity can be expected to have a nonlinear impact on queuing and memory system performance, this section extends the model above for heterogeneous workloads. Results in Section 5 will show that modeling heterogeneity is critical for achieving accurate results.

Three features are needed in order to model heterogeneous processor behavior. First, new model inputs are required. Specifically, each of the model input parameters in Table 2 is measured for each processor. Also, modeling memory access nonuniformity requires additional parameters that specify, for each processor and type of remote memory request, the probability that the request will be directed to each other node.

Second, we require a new model of the varying number of outstanding requests, $M$, that are issued by the processor 
TABLE 5

Accuracy of Processor Throughput Estimates for Weighted Sum versus Average M

\begin{tabular}{|r|r|r|r|r|r|r|r|r|r||r|r|r|}
\hline application & $f_{1}$ & $f_{2}$ & $f_{3}$ & $f_{4}$ & $f_{5}$ & $f_{6}$ & $f_{7}$ & $f_{8}$ & $M_{a v e}$ & weighted & ave M & actual \\
\hline erle16 & .65 & .17 & .09 & .08 & 0 & 0 & 0 & 0 & 1.64 & 1.35 & 1.40 & 1.45 \\
fft-opt16 & .42 & .17 & 0 & 0 & .34 & 0 & 0 & 0 & 2.72 & 1.53 & 1.61 & 1.58 \\
fft16 & .53 & .47 & 0 & 0 & 0 & 0 & 0 & 0 & 1.47 & 1.46 & 1.46 & 1.39 \\
lu-opt8 & .12 & .06 & .06 & .06 & .06 & .06 & .07 & .49 & 5.83 & 2.46 & 2.19 & 2.41 \\
lu8 & .51 & .49 & 0 & 0 & 0 & 0 & 0 & 0 & 1.50 & 1.96 & 1.91 & 1.91 \\
radix8 & .99 & .01 & 0 & 0 & 0 & 0 & 0 & 0 & 1.01 & 1.76 & 1.74 & 1.64 \\
water16 & .73 & .25 & .01 & 0 & 0 & 0 & 0 & 0 & 1.33 & 1.87 & 1.62 & 1.74 \\
\hline
\end{tabular}

before the processor blocks waiting for a memory system response. The SM-ILP model computes system throughput for each possible value of $M$ with the same value of $M$ at every processor and, then, computes a weighted sum of these throughputs, where the weights are computed from the measured distribution of $M, f_{M}$. This weighted sum approach may be valid if the processors generally have the same value of $M$ at the same time, as might be true for the homogeneous SPMD applications that were modeled in [17]. However, the weighted sum technique is difficult to apply in the case where different nodes have different distributions of $M$. Computing the throughput for every possible combination of $M$ values at the different processors, as well as computing the weighting factor for each such throughput, is prohibitively complex. An alternate approach is to use the average value of $M$ at each node. The drawback of using the average value is that it may fail to capture the nonlinear effects of varying $M$; thus, it may not capture a broad distribution of $M$ accurately.

In Table 5, we compare the accuracy of the estimated processor throughput for the weighted sum approach against simply solving the model once for the average value of $M$ for some of the homogeneous applications that were simulated using RSIM. These results provide some evidence that, for current window sizes and applications, the average $M$ approach achieves similar accuracy to the weighted sum approach. We compute average $M$ from the measured $f_{M}$, where the $f_{M}$ are measured assuming an infinite number of MSHRs, and we allow each processor to have its own average value of $M$ which is limited by the number of MSHRs, $M_{h w}$.

The third change is to modify the model equations [18], [16] to compute performance metrics at each resource within the memory system for heterogeneous processor loads. With homogeneous behavior, it is only necessary to compute performance metrics for a single generic resource of type $i$ and a single generic processor load; the total utilization of the particular memory resource, for example, can then be obtained simply by multiplying by the number of processors. This leads to model complexity on the order of the number of types of resources, as was seen in the equations in Section 4.1. For heterogeneous processor loads, in contrast, each processor may have differing memory referencing behavior and, thus, may contribute to differing extents to utilization and contention at each resource. Computing the $N^{2}$ interactions of each processor on each memory system resource increases the complexity of the model equations by a factor of $N^{2}$, which leads to a key question about whether the iterative model will converge in practice. This issue is addressed in Section 5. Efficient coding methods (for both the homogeneous and heterogeneous model) limit the size of the model (measured in $\mathrm{C}++$ code), though, to only about twice that of the homogeneous model. The accuracy improvements that may be obtained by accurately modeling heterogeneous memory request behavior when it exists, rather than assuming homogeneity, are also illustrated in Section 5.

At a high level, the heterogeneous equations have a form similar to the homogeneous equations. Terms require extra indices (given in brackets) to indicate the node of the customer and/or the destination. Thus, a utilization term such as $U_{d c_{\text {loc }}}$ (the mean utilization of the local DC) becomes $U_{d c_{b c}}[i]$ (the mean utilization of the DC of node $i$ by local customers) to reflect the fact that the utilization of the local DC is different for different nodes. The probabilities of the transaction types (e.g., local read or 4-hop write) use an index in a similar fashion. For example, we now have that the probability of transaction yat node $i$ is $P_{y}[i]$. So, the total mean residence time for a customer from node $i, R[i]$, is equal to the sum of its mean residence times at the processor (pe), buses, network, and directory controllers (dc).

$$
R[i]=R_{p e}[i]+R_{b u s}[i]+R_{\text {net }}[i]+R_{d c}[i] .
$$

Examining the DC portion of this equation highlights the differences in the equations between the heterogeneous and homogeneous models. Mean DC residence time is equal to the mean residence time at the local DC plus the mean residence time at the DCs of the other nodes.

$$
R_{d c}[i]=R_{d c_{\text {loc }}}[i]+\sum_{\substack{j \\ j \neq i}} R_{d c_{\text {rem }}}[i][j] .
$$

Only focusing on the mean residence time at the remote $\mathrm{DC}$, it is the sum of the mean residence times over the different types of transactions, denoted by a subscript of $y$.

$$
R_{d c_{r e m}}[i][j]=\sum_{y} R_{d c_{r e m, y}}[i][j] .
$$

The mean residence times of individual transaction types are equal to the probability of the transaction type $\left(P_{y}[i]\right)$ times the visit count $\left(V_{d c_{\text {remy }}}[i][j]\right)$ times the sum of the mean waiting time $\left(W_{d c_{r e m}}[i][j]\right)$ and the service time at the DC $\left(S_{d c}\right)$ :

$$
R_{d c_{\text {rem }, y}}[i][j]=P_{y}[i] V_{d c_{\text {remy }}}[i][j]\left(W_{d c_{r e m}}[i][j]+S_{d c}\right) .
$$


All of the terms in the above equation are inputs, except for the waiting times. The equation for mean waiting time at a remote directory is as follows:

$$
W_{d c_{\text {rem }}}[i][j]=W_{d c_{\text {rem }}}^{\text {others }}[i][j]+W_{d c_{\text {rem }}}^{\text {rem }}[i][j] .
$$

$W_{d c_{\text {crem }}}^{\text {others }}[i][j]$ is the mean waiting time of a node $i$ customer at the DC of node $j$ due to traffic from all nodes other than node $j . W_{d c_{\text {rem }}}^{\text {rem }}[i][j]$ is the mean waiting time of a node $i$ customer at the DC of node $j$ due to traffic from node $j$. Only breaking down $W_{d c_{r e m}}^{\text {rem }}[i][j]$ further, we have that

$$
W_{d c_{r e m}}^{r e m}[i][j]=\sum_{y}\left(W_{d c_{r e m}}^{r e m, y}[i][j]\right) .
$$

The following equations are for the mean waiting times of specific transaction types. Thus, $W_{d c_{r e m}}^{\text {rem, }}[i][j]$ is the mean waiting time by a node $i$ customer at node $j$ 's DC due to node $j$ traffic for transactions of type $y$. Mean waiting time

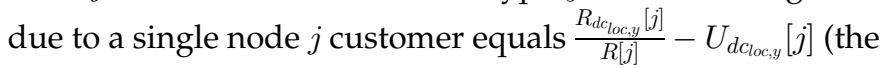
probability that a customer is in the queue but not in service) times the service time plus $U_{d c_{\text {loc } y}}[j]$ (the probability that a customer is in service) times the mean residual life of a customer in service. Therefore, to get the total mean waiting time, we multiply by the number of node $j$ customers, $M[j]$.

$$
\begin{aligned}
& W_{d c_{r e m}}^{r e m, y}[i][j]= \\
& M[j]\left[\left(\frac{R_{d c_{l o c, y}}[j]}{R[j]}-U_{d c_{l o c, y}}[j]\right) S_{d c}+U_{d c_{l o c, y}}[j]\left(\frac{S_{d c}}{2}\right)\right] .
\end{aligned}
$$

Last, we have the equation for the mean utilization of node $i$ 's DC by a local customer.

$$
U_{d c_{l o c, y}}[i]=\frac{P_{y}[i]}{R[i]}\left(V_{d c_{l o c, y}}[i] S_{d c}\right) .
$$

Modeling the other resources in the system is similar to what has been shown here for the DC. All of the details of the heterogeneous AMVA equations can be found in [16].

\subsection{Modeling Bursty Memory Requests and Lock Contention}

In this section, we present two further model extensions. In Section 4.3.1, we adapt the new AMVA techniques proposed in [5] to model bursty memory request traffic observed in the SimOS workload measures. In Section 4.3.2, we develop a method for computing lock synchronization from basic model input parameters.

\subsubsection{Burstiness}

In the previous SM-ILP model, the mean residual line of a "customer in service" at the processor (i.e., a memory request about to be generated) is computed using an intuitively motivated ad hoc interpolation. In this paper, we instead employ a new and significantly more accurate AMVA technique (called "AMVA-decomp") [5] for computing mean residence time at the processor queue. In addition, we adapt the new AMVA techniques in [5] for computing the mean wait at the "downstream" queue (i.e., the local DC in the SimOS architecture) which has bursty arrivals from the local processor and thus increased average queuing delay compared with the random arrivals assumed in the standard AMVA equations.

The use of these new simple AMVA techniques is motivated by the fact that they represent a very favorable balance between accuracy, efficiency, and robustness. Most importantly, they are based on a small number of input parameters for which reliable values are relatively easy to obtain. Furthermore, the solution method is easy to implement and does not add appreciable complexity to the overall AMVA model solution. Finally, the technique is shown in [5] to have very high accuracy over a wide range of system parameters, including parameter values for which it might be expected to have high inaccuracy. More detailed models of the processor service times, with correspondingly more detailed models of the DC arrival process, could be constructed, but such models would require more detailed input parameters that would be more difficult to estimate reliably. The more detailed model would also be more complex to implement and, thus, would only be justified if an appreciable increase in model accuracy could be expected. However, mean delays and system throughput for closed systems are not sensitive to the details of the service distributions (i.e., higher moments of the distribution than the first or second moment) at the various resources in the system [9], [10]. Thus, a more detailed model of the processor service time distribution is not desirable. Validation results later in the paper confirm that, for the purposes of computing system throughput for alternative memory system architectures, the simple AMVA techniques outlined below capture the bursty behavior in sufficient detail to predict system throughput quite accurately.

The AMVA-decomp technique assumes that the server that has the bursty departures (i.e., the processor nodes in the SimOS architecture) can be modeled with a 2-stage hyperexponential distribution of service times. That is, with probability $p$, a given customer has a "small" mean service time, $\tau_{a}$, and, with probability $1-p$, the customer has a "large" mean service time, $\tau_{b}$, where $\tau_{a}<\tau_{b}$ and $\tau=p \tau_{a}+(1-p) \tau_{b}$. The key question in applying this technique for the processors in the heterogeneous model is how to obtain the parameters of a suitable hyperexponential distribution. Two constraints on the distribution are the measured mean service time $(\tau)$ and the coefficient of variation in the service time $\left(C V_{\tau}\right)$. However, this is an underconstrained problem. To apply this technique to modeling heterogeneous bursty processors, we define a third parameter for each processor, $\tau_{a}$, that is equal to the minimum measured value of $\tau .^{3}$ Using $\tau$, $C V_{\tau}$, and $\tau_{a}$, we solve for $\tau_{b}$ and $p$.

In the model of bursty requests at the downstream queues, there are bursts of arrivals and intervals between bursts in which there are no arrivals. This scenario is characterized by three parameters:

3. Note that other choices of $\tau_{a}$, such as a small constant times the minimum measured value of $\tau$, are also possible. What is needed is a value that is approximately correct. We choose to set $\tau_{a}$ to a measured value and allow $\tau_{b}$ to be computed from $\tau_{a}$ because the best value of the "small" mean is likely to be near the measured minimum value of $\tau$, while there is no measured value that corresponds well to $\tau_{b}$. Moreover, the model is more sensitive to the value of $\tau_{a}$ than to that of $\tau_{b}$, especially in the high variance cases where $\tau_{a}<<\tau_{b}$. 
- $\quad k$, the average number of customer arrivals within a burst,

- $I_{i}$, the mean interarrival time within a burst, and

- $I_{o}$, the mean time between bursts.

In applying the bursty request model to the local DCs in the SimOS architecture, the key question again is how to map the requisite parameters to observable quantities in the system. There are two constraints in determining values for these three parameters. First, the coefficient of variation in the interarrival time is determined by the coefficient of variation in the service time at the processor. Second, the throughput at the downstream queue, and thus the mean interarrival time, is determined during the AMVA solution. We create a third constraint by setting $I_{i}$ equal to the value of $\tau_{a}$ since it is reasonable to assume that interarrival time during a burst would be similar to the value of a short service time at the processor. We also assume that downstream burstiness is only caused by requests from the local processor. The superposition of requests from other processors will, on average, be less bursty.

Solving the model with the burstiness equations initially led to some convergence problems. Ensuring that the model converges requires some careful choices of initial values and bounds on the input parameters to make sure that values produced during the iterative solutions are reasonable. For example, $k$ cannot be allowed to be larger than the total number of local customers.

\subsubsection{Lock Contention}

The previous SM-ILP model measured average lock waiting times, which are affected by the memory system architecture, instead of computing these performance measures from more basic parameters that are independent of the memory system architecture. In this paper, lock synchronization effects are computed from basic inputs with a separate lock contention model.

Contention for a particular lock is most naturally modeled by a queue in which the server is the lock and the service time is the lock holding time. The challenge in constructing this queue for the memory system architecture workloads is that the customers (i.e., application processes) queue for the lock while in service at the processor. Furthermore, while holding the lock, the customer may queue for memory system resources and then for further use of the processor. In addition, the program can release the lock while still holding the processor and it can complete service at a memory system resource or processor while still holding the lock. To model these various behaviors, we initially assume that only one lock is held at a time and then relax this assumption.

Lock contention is modeled in a separate queuing network with one queue per lock that has nonnegligible contention and a delay center which represents mean time between releasing a lock and requesting another lock. An example lock queueing model is shown in Fig. 5. $N$ customers in the network represent the processors that are vying for the locks. The parameters for mean time at the delay center and mean service time at the lock queue (i.e., mean time the lock is held) are computed from the memory architecture model.

The basic parameters used to characterize lock behavior in the example are the following:

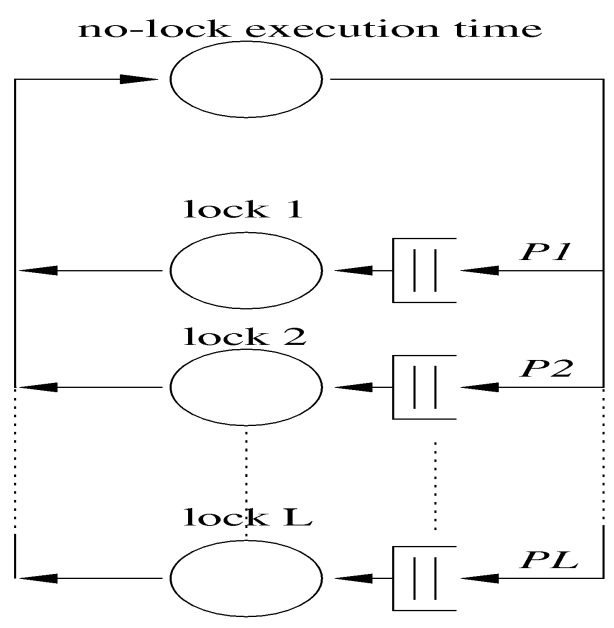

Fig. 5. Example lock contention model.

- $\quad L$ : number of locks that have nonnegligible contention

- $r_{\text {nolock }, j}$ : average number of memory accesses made by processor $j$ between lock requests

- $\quad P_{\text {lock }, j}$ : probability that a lock request from processor $j$ is for lock $i$

- $r_{l o c k_{i}, j}$ : average number of memory requests by processor $j$ while holding lock $i$

To incorporate the effects of lock contention in the architecture model, we iterate between the memory architecture model and the lock contention model. The mean service time at each lock queue is set equal to $r_{l o c k}, j$ times the mean total round trip time for processor $j$ in the memory architecture model $(R[j])$. The mean time at the delay center in the lock contention model for customer $\mathrm{j}$ is set equal to $r_{\text {nolock, } j}$ times $R[j]$. The mean service time at the processor in the architecture model is inflated (i.e., increased to $\tau_{j}+\tau_{j} / r_{\text {nolock }, j}$ ) to include mean lock waiting time computed from the lock model. The iterative solution again increases the complexity of the model, an issue that will be addressed in Section 5. It also causes solution time to increase slightly, but solution time is still on the order of seconds. Moreover, this iterative technique can be generalized for specific cases of nested lock requests by having a separate lock contention model for the locks at each level of the lock hierarchy and iteratively solving the lock models along with the architecture model. The details of the lock contention equations can be found in [16].

\section{Model Validation}

In this section, we present the results of validation experiments that assess the accuracy of the analytic model that is developed in this paper. The validations are performed against SimOS, using SimOS's detailed MXS processor simulator and its NUMA memory system simulator. SimOS runs IRIX 5.3 and all benchmark results include OS behavior that occurred while the benchmark was running. Thus, we measure analytic model inputs and estimate system performance for the complete system behavior instead of for the application alone.

The validation experiments include FFT, LU, Radiosity, and Radix from the Splash-2 suite [23]. Table 6 shows the data sets used for each application. We attempted to obtain 
TABLE 6

Benchmark Data Sets

\begin{tabular}{|l|l|}
\hline app & input size \\
\hline FFT & $-16-\mathrm{n} 1024$ \\
LU & $512 \times 512$ array, 16x16 blocks \\
Radiosity & -batch \\
Radix & $1 \mathrm{M}$ integers, radix 1024 \\
\hline
\end{tabular}

SimOS results for the rest of the Splash-2 benchmark suite, but these applications would not run successfully on the version of the SimOS MXS processor simulator used in this study. (This version of the MXS simulator was one of the first versions to be released for use outside of the research group that developed and initially used the simulator for architectural studies. Thus, various steps needed to get the other applications to run may have been missing from the available documentation.) Similarly, we were unable to make this version of the SimOS MXS simulator produce results for greater than eight processors. Although the number of benchmarks that ran successfully is small, the memory access characteristics captured in the model input parameters vary greatly across these applications as well as in the different periods between barriers in a given application and, thus, the analytical model is exercised over a nontrivial region of the input parameter space. Table 3 and Table 5 illustrate some of the variety in the memory request behavior across the applications. As we will show later, the processor throughput varies from 0.1 to 2.4 instructions per cycle across the application barriers against which we were able to validate, indicating that the differences in memory request behavior among these benchmarks is significant. The low processor throughput estimates also indicate that, although the number of processors is relatively small, significant contention occurs in the memory system (particularly at the directory controllers) and, thus, the ability of the analytic model to

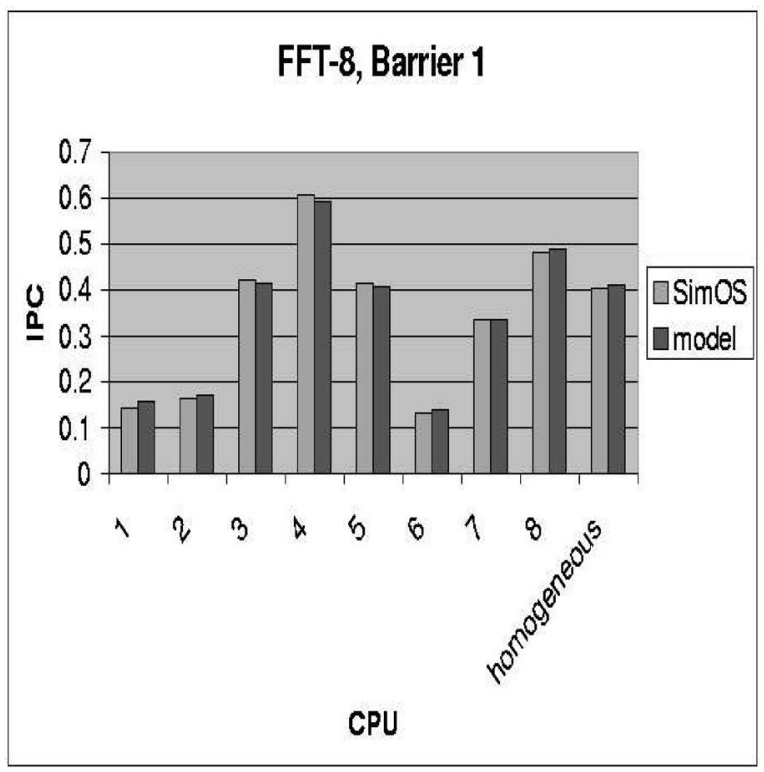

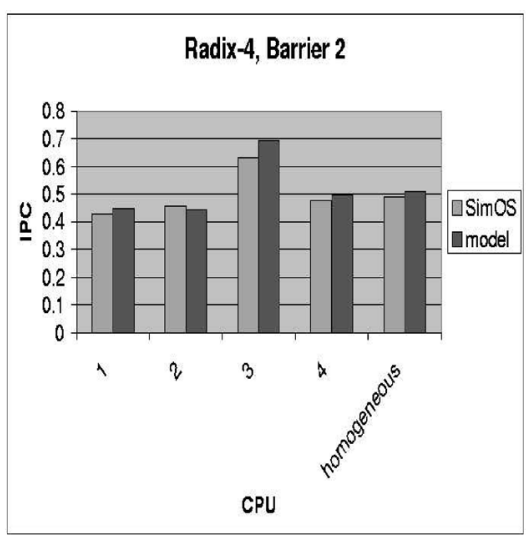

Fig. 7. Validation of Radix-4, barrier 2.

accurately estimate queuing delays is also exercised. This is confirmed by the measured mean queueing delays reported by SimOS for these applications (with the architectural parameters in Table 1).

The validation results for model input parameter values that exhibited the greatest degrees of heterogeneity in processor performance are shown in Fig. 6, Fig. 7, and Fig. 8. These results are for specific barriers (i.e., interbarrier phases) of FFT, Radix, and Radiosity running on 8-node and 4-node versions of SimOS. Each graph gives the throughput (in IPC) for each processor estimated by the new heterogeneous system model as well as the average throughput estimated by the homogeneous model. Results for other barriers of the FFT, LU, Radix, and Radiosity benchmarks (both 8-node and 4-node) are presented in Table 7. The column numbers in the table correspond to node numbers. For each pair of rows, the first row is the IPC reported by SimOS and the second row is the IPC predicted by the model. The rightmost column corresponds to a homogeneous model using the average statistics, where the first row is the average IPC across all nodes reported by SimOS and the second row is the IPC predicted by the previous homogeneous model using input parameters that are averaged across all nodes.

These results show that the analytic estimates of per processor throughput agree quite closely with the SimOS measurements, even when each processor throughput is remarkably different. The model achieves accurate perfor-

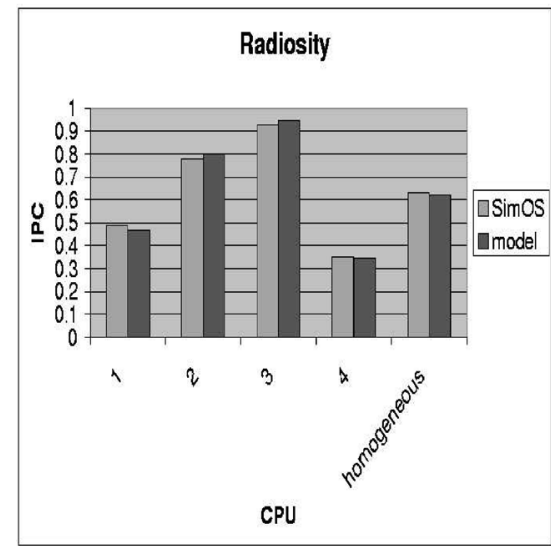

Fig. 8. Validation of Radiosity-4. 
TABLE 7

Validation Results: Processor Throughput (IPC) Estimates

\begin{tabular}{|l|r|r|r|r|r|r|r|r|r|}
\hline app & 1 & 2 & 3 & 4 & 5 & 6 & 7 & 8 & average \\
\hline FFT-4 (b1) & 0.12 & 0.14 & 0.15 & 0.17 & & & & & 0.15 \\
& 0.11 & 0.13 & 0.15 & 0.17 & & & & & 0.16 \\
\hline \hline FFT-4 (b2) & 0.68 & 0.73 & 1.06 & 0.91 & & & & & 0.82 \\
& 0.61 & 0.66 & 0.99 & 0.82 & & & & & 0.74 \\
\hline \hline FFT-4 (b3) & 0.47 & 0.48 & 0.55 & 0.62 & & & & & 0.52 \\
& 0.43 & 0.43 & 0.50 & 0.57 & & & & & 0.47 \\
\hline \hline FFT-8 (b1) & 0.14 & 0.16 & 0.42 & 0.61 & 0.41 & 0.13 & 0.33 & 0.48 & 0.40 \\
& 0.16 & 0.17 & 0.41 & 0.59 & 0.41 & 0.14 & 0.33 & 0.49 & 0.41 \\
\hline \hline FFT-8 (b3) & 0.52 & 0.56 & 0.52 & 0.53 & 0.39 & 0.43 & 0.47 & 0.50 & 0.50 \\
& 0.51 & 0.55 & 0.55 & 0.52 & 0.39 & 0.42 & 0.48 & 0.49 & 0.50 \\
\hline \hline LU-4 (b2) & 1.27 & 0.59 & 0.59 & 1.43 & & & & & 0.82 \\
& 1.40 & 0.61 & 0.62 & 1.37 & & & & & 0.91 \\
\hline \hline LU-4 (b3) & 2.17 & 1.79 & 1.92 & 2.22 & & & & & 1.96 \\
& 2.37 & 1.88 & 2.06 & 2.50 & & & & & 2.13 \\
\hline \hline LU-4 (b4) & 0.85 & 1.09 & 0.81 & 1.20 & & & & & 1.06 \\
& 0.89 & 1.14 & 0.72 & 1.22 & & & & & $\mathbf{1 . 3 2}$ \\
\hline \hline Radix-4 (b2) & 0.43 & 0.46 & 0.63 & 0.48 & & & & & 0.49 \\
& 0.45 & 0.44 & 0.69 & 0.50 & & & & & 0.51 \\
\hline \hline Radix-4 (b6) & 0.30 & 0.36 & 0.26 & 0.45 & & & & & 0.36 \\
& 0.32 & 0.39 & 0.26 & 0.48 & & & & & 0.39 \\
\hline \hline Radix-8 (b1) & 0.64 & 0.64 & 0.62 & 0.64 & 0.61 & 0.61 & 0.61 & 0.63 & 0.62 \\
& 0.68 & 0.64 & 0.61 & 0.64 & 0.62 & 0.61 & 0.60 & 0.63 & 0.62 \\
\hline \hline Radix-8 (b3) & 0.90 & 0.70 & 0.68 & 0.64 & 0.56 & 0.50 & 0.42 & 0.45 & 0.69 \\
& 0.87 & 0.67 & 0.67 & 0.63 & 0.58 & 0.52 & 0.47 & 0.46 & 0.69 \\
\hline \hline Radiosity-4 (b9) & 0.49 & 0.78 & 0.93 & 0.35 & & & & & 0.63 \\
& 0.47 & 0.80 & 0.95 & 0.34 & & & & & 0.62 \\
\hline
\end{tabular}

mance estimates although memory request behavior is modeled statistically and at a high level of abstraction. As mentioned before, the complexity of the new analytic model makes its tractability a key question. In validating the model, however, we discovered no cases where the model did not converge to a solution within a matter of a few seconds, in spite of strong heterogeneity in the model inputs and in the estimated per processor throughputs.

Although the homogeneous analytic model is also often accurate in estimating the average processor throughput, the new estimates of per processor throughput from the heterogeneous model are crucial to accurately estimating the impact of the memory system architecture on application execution times because, for applications employing barriers, the barrier only completes execution when the slowest processor reaches the barrier. Moreover, there are examples, such as LU-4 barrier 4, for which the homogeneous model is not even accurate with respect to the average node behavior reported by SimOS. This is due to the fact that heterogeneity can have nonlinear effects on queuing delays in the memory system and these nonlinear effects are only captured in the heterogeneous model.

Validation of the lock synchronization modeling was difficult in that none of the benchmarks that succeeded in running on the MXS simulator in our version of SimOS exhibited significant lock contention. Instead, we implemented the "Sieve of Eratosthenes" algorithm for finding prime numbers and found that executing this application on SimOS exhibited significant lock contention. The accuracy of the analytic model with complementary service time inflation for lock contention, as compared with SimOS measures for the application, is illustrated by the results in Fig. 9 and Fig. 10. The application's heavy lock contention is revealed by the high mean lock waiting times reported in Fig. 9. The mean lock waiting times and processor throughputs (measured in memory requests per cycle) estimated by the analytic model agree reasonably well with the measured values, even in this extreme case.

\section{Applications of the Model}

The model developed in this paper can be applied to efficiently obtain initial answers to numerous questions about architectural designs and the interactions between applications and the architecture. Recall that the memory system parameters (below the L2 cache) can be varied to explore the design space without having to rerun the detailed simulator to obtain new input parameters. The model can reveal performance bottlenecks at specific system resources due to heterogeneous and bursty memory request traffic. This section illustrates three applications of the model to such issues, pointing out cases in which the previous homogeneous model provides inaccurate results.

\subsection{Decoupling the Network Interface from the Directory Controller}

As discussed in Section 2, the SimOS architecture requires all traffic into and out of a node to pass through the 


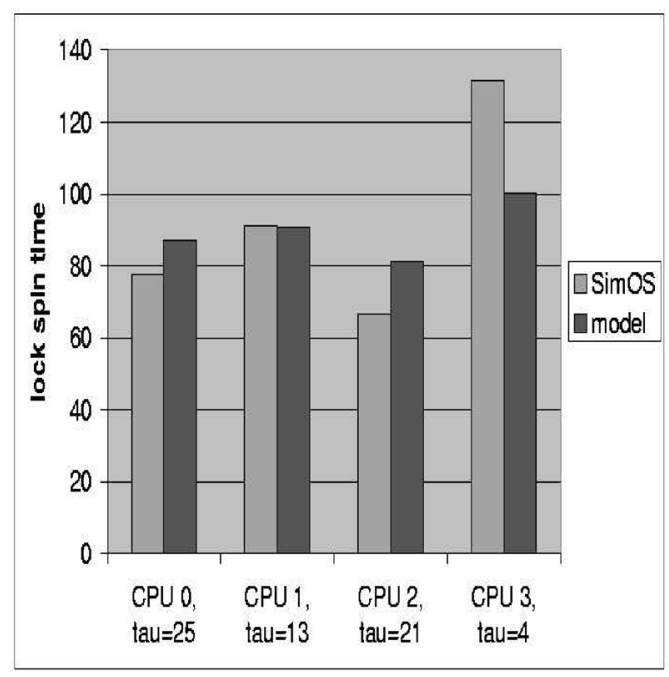

Fig. 9. Validation of lock contention estimates ("Sieve of Eratosthenes" algorithm on four CPUs).

directory controller (DC). The DC effectively assumes the responsibility of being the network interface (NI) for all traffic, including traffic that does not require use of the directory. This coupling of the DC and NI may, in some cases, create a bottleneck, especially as processor speed increases relative to memory speed.

An interesting architectural question that can be quickly assessed with the model is the performance gain that could be achieved by decoupling the NI from the DC, given that the $\mathrm{DC}$ is twice as slow relative to the processor speed as compared to the default values in Table 1. Fig. 11 shows that, for an 8-way parallel execution of Radix (barrier 2), decoupling the NI and the DC reduces the cycle count of the barrier from $163 \mathrm{k}$ to $124 \mathrm{k}$ and moves the bottleneck from CPU 3 to CPU 7.

The rightmost pair of bars shows the performance impact predicted by the homogeneous model and there are two key inaccuracies worth noting. Quantitatively, the homogeneous model predicts barrier cycle counts 30 percent less than those predicted by the heterogeneous model.

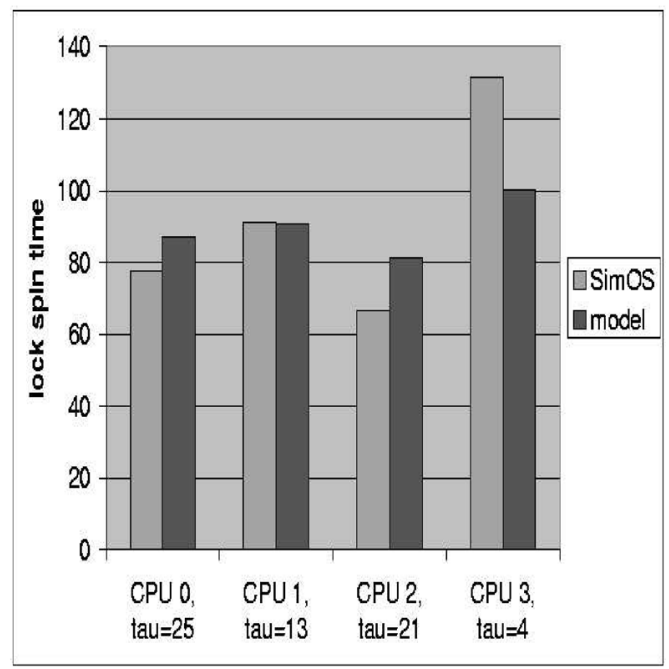

Fig. 10. Validation of processor throughput estimates with lock contention ("Sieve of Eratosthenes" algorithm on four CPUs).

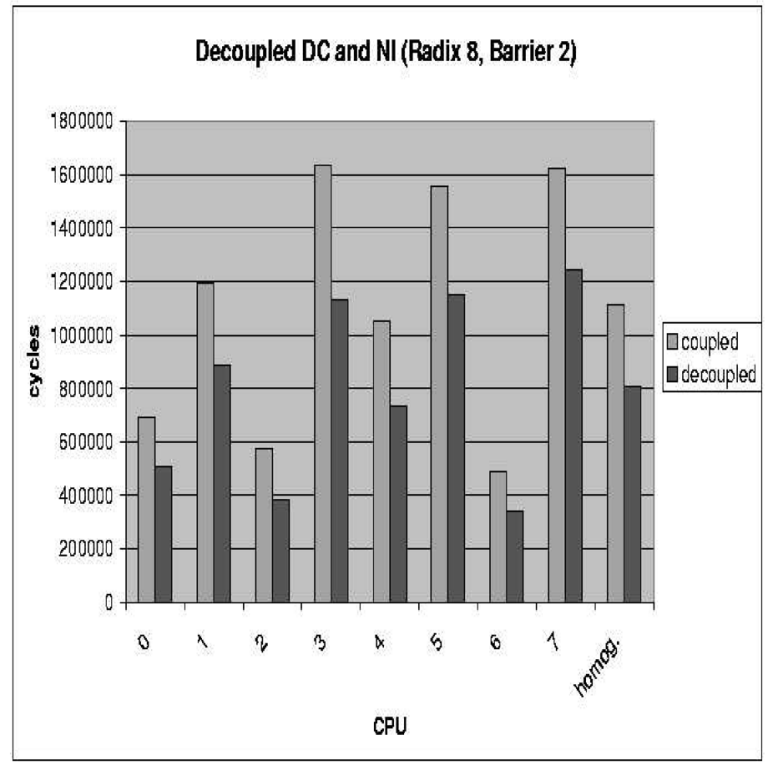

Fig. 11. Impact of decoupling DC and NI.

Qualitatively, the homogeneous model fails to capture the shift in the bottleneck.

\subsection{Importance of Data Layout}

The model input parameter values for a given application or workload can be useful in identifying opportunities for tuning the application (or kernel). For example, the input parameters that characterize where memory requests from each processor are directed reveal insight into data layout issues. More specifically, good data layout schemes maximize the fraction of requests that can be serviced locally. That is, for a given application, if one layout scheme has a higher probability of servicing a memory request locally than another scheme, then it will likely have better performance since local requests have lower latency than remote requests.

For barrier 3 of LU-4, Fig. 12 illustrates the performance gains that can be achieved for a hypothetical 50 percent increase in the probability that a request is satisfied locally. Of interest is the reduction in execution time that is achieved by the hypothetical tuning at the bottleneck processor (i.e., CPU 3 in Fig. 12). The magnitude of the decrease in execution time guides how much effort should be expended in looking for opportunities to increase data locality in the LU code.

As shown in Fig. 12, the homogeneous model predicts a decrease in average processor execution time that is similar in magnitude (although larger in percentage) to the decrease in execution time for the bottleneck processor. However, the decrease in average processor execution time is not generally a reliable estimate for what will occur at the bottleneck center.

\subsection{Tuning the Operating System}

As discussed in Section 3.3, the operating system causes the memory request heterogeneity that is observed in workloads with applications that are generally considered homogeneous. For example, Table 4 shows that barrier 2 of FFT- 4 is heterogeneous in $\tau$ because of the kernel. To determine the performance gain that could be achieved by 


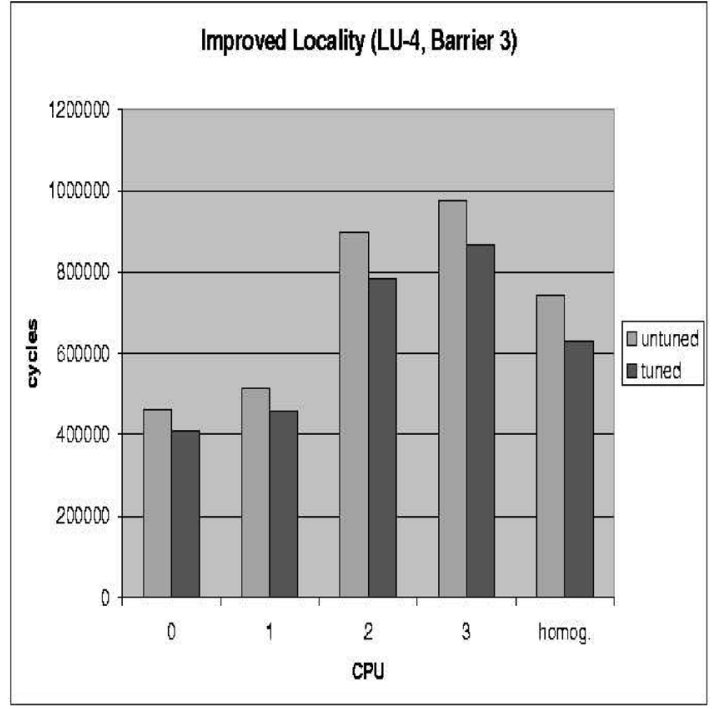

Fig. 12. Performance impact of improved memory locality.

hypothetically tuning the kernel for homogeneity, we compared the performance of this barrier against the performance of the same barrier with homogeneous kernel behavior. The OS parameters at each node are assumed to be equal to their averages across all four nodes. Fig. 13 reveals that if the OS can be tuned for greater uniformity in processor usage within the time that the application executes between barriers rather than on a much coarser time scale, this would lead to a 20 percent reduction in cycle count for this barrier. Clearly, this study could not have been performed without a heterogeneous model.

\section{Conclusions}

We have developed and validated a new analytical model for evaluating the performance of shared memory multiprocessors with ILP processors and heterogeneous processor workloads. This work extends prior research in this area in three ways: 1) adapting and validating the model for a different architecture than that considered in previous work, 2) modeling heterogeneous node behavior that was reported by SimOS even when running homogeneous applications, and 3) applying new techniques for modeling bursty memory requests and developing techniques for modeling lock synchronization. Despite the complexity of modeling processor heterogeneity and nonuniform memory access probabilities, bursty memory traffic, and lock contention, the model converges quickly, is still several orders of magnitude faster to solve than detailed simulation, and the number of input parameters remains manageable. The model validates extremely well for individual processor throughput estimates over a range of Splash-2 benchmarks that have a wide variety of memory request behaviors, which leads to a wide range of observed processor throughputs. Examples in Section 6 show how the model can be used to study architectural design issues as well as to study interactions between the architecture and the application. Moreover, the examples show that insight can be gained simply from looking at the input parameter values that are measured for a particular workload. Model parameters reveal application behavior and, in turn,

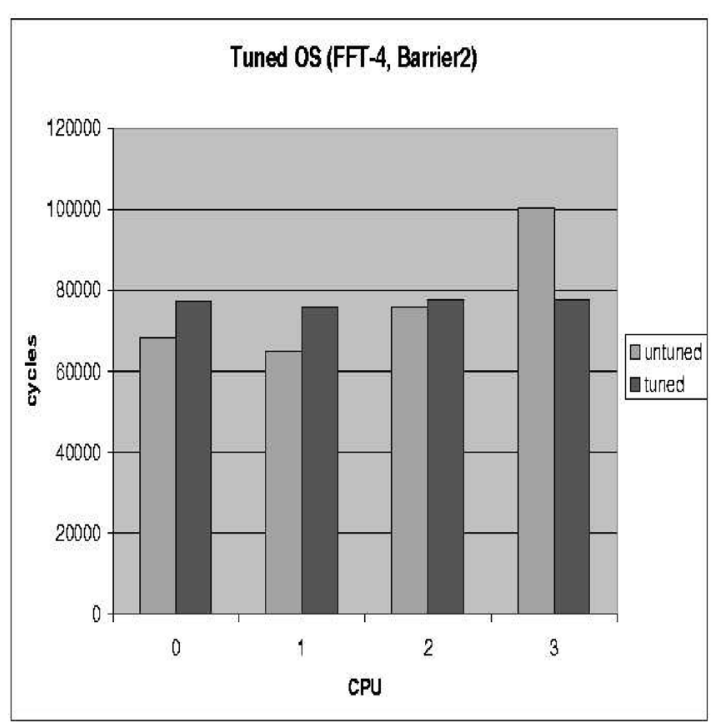

Fig. 13. Predicted impact of tuning the OS.

opportunities for architectural and application optimizations that target the bottlenecks revealed by the behavior (such as not enough parallelism in the requests issued to memory).

The model is being made available for use by others in the POEMS environment [1]. The real test of the model is how it performs in a commercial architecture design context for which public data is unavailable. Perhaps in making the model available to commercial systems designers, feedback can be obtained about its accuracy in a real system design setting. Results for systems with more nodes would also be interesting since the model's speed advantage over simulation would be even greater. Future research topics also include investigating methods of coupling the architectural model with a more abstract model of the communication and synchronization behavior in the application.

\section{ACKNOWLEDGMENTS}

This research is supported, in part, by the US Defense Advanced Research Projects Agency/ITO under Contract N66001-97-C-8533, the US National Science Foundation under Grants MIP-9625558, EIA-9971256, EIA-9975024, and EIA-0127857, and by the Natural Sciences and Engineering Research Council of Canada under Grant OGP-0000264.

\section{REFERENCES}

[1] V. Adve, R. Bagrodia, J. Browne, E. Deelman, A. Dube, E. Houstis, J. Rice, R. Sakellariou, D. Sundaram-Stukel, P. Teller, and M. Vernon, "POEMS: End-to-end Performance Design of Large Parallel Adaptive Computation Systems," IEEE Trans. Software Eng., vol. 26, no. 11, pp. 1027-1048, Nov. 2000.

[2] A. Agarwal, M. Horowitz, and J.L. Hennessy, "An Analytical Cache Model," Trans. Computer Systems, vol. 7, no. 2, pp. 184-215, May 1989.

[3] D. Albonesi and I. Koren, "A Mean Value Analysis Multiprocessor Model Incorporating Superscalar Processors and Latency Tolerating Techniques," Int'l J. Parallel Programming, pp. 235-263, 1996.

[4] L.A. Barroso, K. Gharachorloo, and E. Bugnion, "Memory System Characterization of Commercial Workloads," Pro. 25th Ann. Int'l Symp. Computer Architecture, pp. 3-14, June 1998. 
[5] D. Eager, D. Sorin, and M. Vernon, "AMVA Techniques for High Service Time Variability," Proc. ACM SIGMETRICS, pp. 217-228, June 2000.

[6] D. Kroft, "Lockup-Free Instruction Fetch/Prefetch Cache Organization," Proc. Eighth Int'l Symp. Computer Architecture, pp. 81-87, May 1981

[7] J. Kuskin, D. Ofelt, M. Heinrich, J. Heinlein, R. Simoni, K. Gharachorloo, J. Chapin, D. Nakahira, J. Baxter, M. Horowitz, A. Gupta, M. Rosenblum, and J. Hennessy, "The Stanford FLASH Multiprocessor," Proc. 21st Int'l Symp. Computer Architecture, pp. 302-313, Apr. 1994.

[8] J. Laudon and D. Lenoski, "The SGI Origin: A ccNUMA Highly Scalable Server," Proc. 24th Ann. Int'l Symp. Computer Architecture, pp. 241-251, June 1997.

[9] E. Lazowska, "The Use of Percentiles in Modelling CPU Service Time Distributions," Proc. IFIP W.G.7.3 Int'l Symp. Computer Performance Modeling, Aug. 1977.

[10] E. Lazowska, J. Zahorjan, G. Graham, and K. Sevcik, Quantitative System Performance, Computer System Analysis Using Queueing Network Models. Prentice Hall, May 1984.

[11] M. Oskin, F.T. Chong, and M. Farrens, "HLS: Combining Statistical and Symbolic Simulation to Guide Microprocessor Designs," Proc. 27th Ann. Int'l Symp. Computer Architecture, pp. 7182 , June 2000

[12] V. Pai, P. Ranganathan, and S. Adve, "RSIM Reference Manual," Technical Report 9705, Dept. of Electrical and Computer Eng., Rice Univ., Aug. 1997.

[13] V.S. Pai, P. Ranganathan, S.V. Adve, and T. Harton, "An Evaluation of Memory Consistency Models for Shared-Memory Systems with ILP Processors," Proc. Seventh Int'l Conf. Architectural Support for Programming Languages and Operating Systems, pp. 1223, Oct. 1996.

[14] M. Rosenblum, S. Herrod, E. Witchel, and A. Gupta, "Complete Computer System Simulation: The SimOS Approach," IEEE Trans. Parallel and Distributed Systems, vol. 3, no. 4, pp. 34-43, Apr. 1995.

[15] A. Saulsbury, F. Pong, and A. Nowatzyk, "Missing the Memory Wall: The Case for Processor/Memory Integration," Proc. 23rd Ann. Int'l Symp. Computer Architecture, pp. 90-101, May 1996.

[16] D. Sorin, J. Lemon, D. Eager, and M. Vernon, "A Customized MVA Model for Shared-Memory Systems with Heterogeneous Applications," Technical Report 1400, Computer Sciences Dept., Univ. of Wisconsin, Madison, 1999.

[17] D. Sorin, V. Pai, S. Adve, M. Vernon, and D. Wood, "Analytic Evaluation of Shared-Memory Parallel Systems with ILP Processors," Proc. 25th Int'l Symp. Computer Architecture, pp. 380-391, June 1998.

[18] D. Sorin, M. Vernon, V. Pai, S. Adve, and D. Wood, "A Customized MVA Model for ILP Multiprocessors," Technical Report 1369, Computer Sciences Dept., Univ. of Wisconsin, Madison, Mar. 1998

[19] V. Soundararajan, M. Heinrich, B. Verghese, K. Gharachorloo, A. Gupta, and J. Hennessy, "Flexible Use of Memory for Replication/ Migration in Cache-Coherent DSM Multiprocessors," Proc. 25th Ann. Int'l Symp. Computer Architecture, pp. 342-355, June 1998.

[20] B. Verghese, S. Devine, A. Gupta, and M. Rosenblum, "Operating System Support for Improving Data Locality on CC-NUMA Compute Servers," Proc. Architectural Support for Programming Languages and Operating Systems VII, pp. 279-289, Oct. 1996.

[21] D. Willick and D. Eager, "An Analytical Model of Multistage Interconnection Networks," Proc. ACM SIGMETRICS, pp. 192-202, May 1990

[22] S.J.E. Wilton and N.P. Jouppi, "CACTI: An Enhanced Cache Access and Cycle Time Model," IEEE J. Solid-State Circuits, vol. 31, no. 5, pp. 677-688, May 1996.

[23] S. Woo, M. Ohara, E. Torrie, J. Singh, and A. Gupta, "The SPLASH-2 Programs: Characterization and Methodological Considerations" Proc. 22nd Int'l Symp. Computer Architecture, pp. 24-36, June 1995

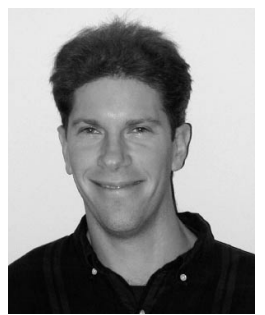

Daniel J. Sorin received the BSE degree in electrical and computer engineering from Duke University in 1996, the MS and PhD degrees in electrical and computer engineering from the University of Wisconsin-Madison in 1998 and 2002, respectively. He is an assistant professor in the Department of Electrical and Computer Engineering at Duke University. Dr. Sorin's research interests are in multiprocessor memory systems, with an emphasis on availability, verification, and analytical performance evaluation. He is a member of the IEEE.

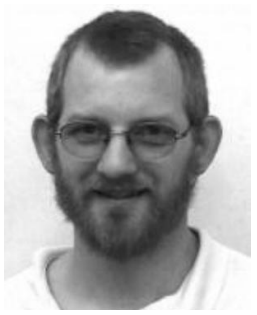

Jonathan L. Lemon received the BSE degree in computer science and electrical engineering from the University of California at Berkeley in 1990 and worked for Hewlett Packard and Amdahl before attending the University of Wisconsin-Madison, where he received the MS degree in computer science in 1999. He left the university in 2000 to cofound a caching company which was subsequently purchased by Cisco Systems, where he is currently employed. In his spare time, he contributes to development of the FreeBSD Operating System, where he is well-known for his invention of the kqueue facility.

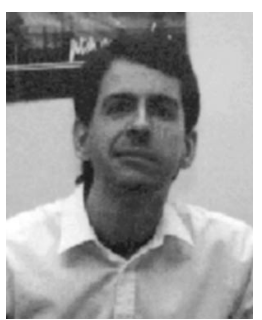

Derek L. Eager received the BSc degree in computer science from the University of Regina, Saskatchewan, Canada, in 1979, and the MSc and $\mathrm{PhD}$ degrees in computer science from the University of Toronto in 1981 and 1984, respectively. Currently, he is a professor in the Department of Computer Science at the University of Saskatchewan. His research interests are in the areas of performance evaluation, streaming media delivery, and distributed systems.

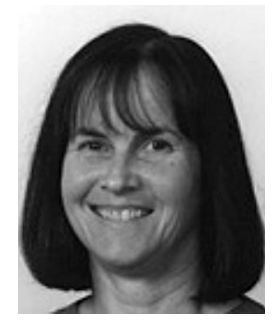

Mary K. Vernon received the BS degree with departmental honors in chemistry and the PhD degree in computer science from the University of California at Los Angeles. In 1983, she joined the Computer Science Department at the University of Wisconsin-Madison, where she is currently a professor of computer science and industrial engineering. Her research interests include performance analysis techniques for evaluating computer/communication system design tradeoffs, parallel/distributed architectures and software systems, computer system security, and streaming media content distribution. Dr. Vernon has served on the editorial board of the IEEE Transactions on Parallel and Distributed Systems, the board of directors of the Computing Research Association, the executive committee of the National Computational Science Alliance, and as chair of the ACM SIGMETRICS. She received a US National Science Foundation Presidential Young Investigator Award in 1985, the ACM Fellow award in 1996, and a University of Wisconsin Vilas Associate Award in 2000. She is a member of the IEEE Computer Society, the ACM, and IFIP WG 7.3 on Information Processing System Modeling, Measurement, and Evaluation.

$\triangleright$ For more information on this or any computing topic, please visit our Digital Library at http://computer.org/publications/dlib. 